Taylor, C. (2016)

\title{
The Language Animal. The Full Shape of the Human Linguistic Capacity
}

Cambridge, MA; Londres: Belknap Press of Harvard University Press, 352 pp.

Because we still have a lot we don't understand in this domain. With phrases like "animal possessing language", we are trying to answer a question like: "what is human nature?" (Taylor, 2016, pp. 338-339)

El lenguaje ha sido objeto y medio central de estudio a lo largo de la historia de la humanidad. ¿Qué significa tener lenguaje? Diversos autores lo han puesto en el centro de sus planteamientos teóricos, a pesar de tener diferencias ontológicas y epistemológicas casi irreconciliables, como es el caso de Wittgenstein y Lacan, por ejemplo. Sobre este complejo tema, Charles Taylor, filósofo de reconocida trayectoria en el estudio de las humanidades y profesor de la Universidad de McGill, consolida su propuesta con una sólida y exhaustiva reflexión desde la hermenéutica, afirmando que el lenguaje solo puede ser entendido si comprendemos el rol constitutivo que tiene en la vida humana.

En el primer capítulo, Taylor presenta dos visiones del lenguaje de manera introductoria. En primer lugar, menciona la perspectiva designativa/ instrumental, HLC (Hobbes, Locke y Condillac), que concibe al lenguaje como un instrumento para representar la realidad de manera fidedigna, basado en la correspondencia exacta entre el signo lingüístico y las ideas u objetos que se intentan describir. Por otro lado, expone la contraparte, HHH (Hamann, Herder y Humboldt), que define el lenguaje como un elemento constitutivo de la realidad humana, en tanto la representa y la crea desde una manera de vivirla.

En el siguiente capítulo, el autor aborda el proceso de adquisición del lenguaje. Recuerda el rol fundamental de la experiencia intersubjetiva en el desarrollo de la capacidad lingüística y de la autoconciencia en los niños. Además, señala que la función de las representaciones no es solo describir, sino crear comunión. El acto del habla es la matriz del lenguaje; su primacía es evidente en los marcadores de los interlocutores y en el uso de diferentes recursos lingüísticos, utilizados en relación con la situación conversacional presente.

En el tercer capítulo, Taylor expone cinco razones por las que el lenguaje es el medio en el que estamos sumergidos, un rasgo de lo que somos, más que un codificador de información. La primera razón radica en que la intersubjetividad es una condición para el desarrollo del lenguaje. En segundo lugar, la 
conciencia lingüística implica un sentido del todo, aquí entra el concepto de Wohnen en Heidegger: la cosa representada no es la entidad objetivada por un solo significado, sino el conjunto de significados híbridos que la cosa alberga en diferentes contextos. Además, la articulación del significado humano no solo se codifica mediante palabras, sino por medio de acciones y repertorios conductuales, pues los gestos pueden cambiar radicalmente el significado de una expresión. En cuarto lugar, el despliegue de significados implica más que una palabra correcta; abarca también la comunicación no verbal. Por último, este despliegue o revelación tiene una fuerza constitutiva en los hechos sociales: los establece, sostiene y transforma, son hechos ontológicamente lingüísticos. Considerando estas razones, el modelo de Saussure no termina de entender la creación del significado humano; en muchos casos, no hay una relación arbitraria entre el significante y el significado, por lo que no están necesariamente diferenciados.

A continuación, en el capítulo cuarto, Taylor presenta exhaustivamente la perspectiva instrumentalista sobre el lenguaje. Empieza por los postulados iniciales de Locke y Hobbes, quienes plantean que el surgimiento de ideas antecede al hecho lingüístico y, por lo tanto, el lenguaje asocia ideas con términos de manera arbitraria. Luego desarrolla la perspectiva de Condillac: el lenguaje nos permite tener empire sur notre imagination, que no es sino la capacidad de controlar nuestra atención. Después, reconoce el aporte principal de Frege: estudiar el rol del significado como producto de la suma de palabras que apuntan a un referente, diferenciándolo claramente de los procesos implicados en su elaboración, dejando atrás el psicologismo y sus explicaciones normativas. Esto da origen a las reflexiones de la filosofía analítica, como una corriente que estudia las relaciones lógicas entre los diferentes enunciados proposicionales. Afirma que este modelo es objetivista, pues propone una ontología del lenguaje basada en la neutralidad, cuyas condiciones de verdad deben ser expresadas en un vocabulario fisicalista desde la tercera persona, y lo que está fuera de estos márgenes no existe ni añade nada nuevo a la comprensión de la realidad. Cierra el capítulo afirmando que no hay nada malo con la semántica objetivista, pero que el lenguaje no puede restringirse a una de sus dimensiones.

En línea con su propuesta constitutiva, en el capítulo quinto, desarrolla la dimensión figurativa del lenguaje para cuestionar profundamente algunos postulados del modelo instrumentalista, como la arbitrariedad de la relación significante-significado y el reduccionismo al lenguaje literal. Comienza por las metáforas puntuales, que emergen inicialmente como un préstamo intencional de términos con cualidades aplicables a realidades más abstractas, para terminar utilizándose automáticamente. Asimismo, propone el concepto de formatos 
estructurales, elementos que configuran la realidad y dan forma a muchas metáforas. Por ejemplo, el formato arriba/abajo: "estoy emocionalmente en el hoyo", "el candidato está en la cima de las encuestas". En estas frases vemos cómo formulamos metáforas para representar el crecimiento y el bienestar con el "arriba", y las realidades opuestas con el "abajo". Por otra parte, encontramos a los símbolos, cuyo significado es irreducible a términos más básicos; y a los íconos, que no involucran el uso del lenguaje verbal, como los gestos, que incluso crean expresiones lingüísticas. Afirma incluso que la ciencia se funda en formas simbólicas metaforizadas.

En los siguientes capítulos, Taylor desarrolla su posición sobre el lenguaje desde la filosofía hermenéutica, que concibe a los significados como elementos interconectados en redes y constelaciones semánticas complejas, que no se definen desde la objetividad ni desde la linealidad. Dedica varias páginas a explicar la articulación y creación de significados, las dos dimensiones constitutivas del lenguaje, ejes centrales del libro.

En lo referente a la articulación constitutiva de significados, en el capítulo sexto, afirma que es un proceso que involucra descubrimiento y expresión. Plantea la categoría de significados metabiológicos, contenidos que no son necesarios para la supervivencia, pero que tienen sentido por su significancia práctica en la experiencia humana, que solo existen en la expresión lingüística. Expone que, en el terreno moral, los principios e ideales están fundados en el entendimiento hermenéutico de nuestras emociones, que puede ser confirmado mediante intuiciones individuales o intuiciones colectivas presentes o pasadas.

Otra manera de articular significados se encuentra en la corporeización y actuación de significados, comportamientos o patrones conductuales que no representan ni significan, sino que son unidades independientes de significados culturales, como, por ejemplo, el comportamiento del típico "macho" latino, pues cuando la persona actúa no está representando al macho; la actuación es el macho. Explica también la semántica del arte, que produce símbolos intraducibles a la prosa descriptiva, pues son retratos, representaciones que revelan un insight distinto sobre la vida humana desde su estructura, por lo que la pieza artística no es un estímulo incondicionado.

No conforme con el carácter constitutivo de la articulación de significados existentes, Taylor va más allá y trabaja, en el capítulo séptimo, la fuerza creadora del discurso, la segunda dimensión constitutiva del lenguaje. Dentro de esta dimensión, propone el concepto de footings, marcadores verbales y no verbales que sostienen la relación entre los interlocutores y los patrones de comunicación, por ejemplo, en vínculos del tipo jefe-subordinado. Además, menciona que el lenguaje tiene una capacidad performativa, pues hay situaciones sociales y 
footings, como el matrimonio o la guerra, que existen a partir de su declaración. Resalta la necesidad que tienen estos hechos de ser renovados y actuados para mantener su significado mediante la práctica social, lo que sucede también con el macroorden social.

Dedica el octavo capítulo a explicar el proceso de producción de significados que se extraen de una narración. Sostiene que las narraciones nos dan un entendimiento único e insustituible de la vida, con insights que solo se obtienen del proceso narrativo. Son Gestalts diacrónicas, no inductivas, pues emergen de la relación entre las intuiciones nuevas y el conocimiento previo a lo largo del tiempo. La historia construye nuestra identidad personal, pues solo en el discurso de la narración llegamos a ser un self. Incluso en el campo moral, sostiene que, aunque compartiésemos valores, las intuiciones e historias que nos llevaron a tenerlos no son las mismas, y estas son una parte del significado del valor.

A la luz de su perspectiva constitutiva, afirma que es posible darle una mirada distinta a la hipótesis de Sapir-Whorf, abordada en el capítulo noveno. Esta hipótesis plantea que el lenguaje moldea el pensamiento y que muchas veces existen visiones inconmensurables, que implican necesariamente inconmensurabilidad entre lenguas diferentes. Si bien acepta que en ciertas áreas es más sencillo llegar a un consenso, como es el caso de los elementos que nos rodean y las concepciones metafísicas, resalta la importancia de considerar las profundas diferencias culturales, ergo, lingüísticas. De esta manera, superamos una visión etnocéntrica, donde todo significado cultural es traducible a la lógica occidental. Para llegar a un dominio completo de la otra lengua, según Taylor, es necesaria la validación y comprensión de significados culturales.

En el capítulo final, Taylor concluye que la capacidad lingüística tiene un rango muy amplio, que supera a la capacidad intelectual. Las acciones expresivas, los retratos y las metáforas dan cuenta de una capacidad lingüística que es esencialmente social, pues surge de una conciencia compartida sobre el mundo. Evita rivalizar el paradigma analítico con el hermenéutico, pues afirma que en la ética, por ejemplo, la razón debe dar un giro hermenéutico. En el cierre del libro, presenta su próximo proyecto, que estudiará el rol de la poesía en la exploración de la naturaleza humana.

Este libro constituye una sólida propuesta hermenéutica para entender la ontología del lenguaje, un tema muy complejo que interesa no solo a la academia, sino a la humanidad en general. Desde el cine, encontramos el caso de Arrival, que nos ofrece un insight distinto sobre las reflexiones filosóficas del lenguaje que trabaja Taylor, específicamente sobre la hipótesis de SapirWhorf, a partir de una historia que relata el encuentro entre la raza humana 
con una población extraterrestre que tiene una concepción no lineal del tiempo, expresada en su lenguaje.

Según Taylor, el lenguaje articula y crea contenidos que se revelan en su misma estructura. ¿El lenguaje tendrá un rol constitutivo en nuestra concepción del tiempo y del espacio? ¿Es posible para una persona que tiene al inglés, alemán o francés como lengua nativa, disgregar el significado del verbo to be, sein o être en los significados de los verbos ser y estar? ¿Podemos unificar nosotros, hispanohablantes, el ser y estar en una sola unidad semántica cuando aprendemos una de estas lenguas? ¿El ser necesariamente necesita un espacio? ¿Concebimos nosotros, hispanohablantes, la posibilidad de la existencia de un ser fuera del espacio?

Las diferencias lingüísticas plantean preguntas apasionantes, que no solo borran los límites de diversas disciplinas, sino que exigen el diálogo entre ellas, pues un abordaje interdisciplinario es casi obligatorio. Es claro, pues, que es en el lenguaje donde nos consolidamos como especie diferenciada y, por lo tanto, los problemas del lenguaje son problemas de todas las actividades humanas.

Fernando García Blesa 